\title{
Mobile Mini-Payment Scheme Using SMS-Credit
}

\author{
Edison Lai \\ Radio Planning Networks Division \\ Hutchison Telephone \\ Macau \\ edisonr@macau.ctm.net
}

\author{
Simon Fong \\ Faculty of Science and Technology \\ University of Macau \\ Macao \\ ccfong@umac.mo
}

\begin{abstract}
Mobile Phone has been widely used in the world and adopted in different application areas involved with SMS has been evolved for downloading logo, ring-tones, advertisement, security notes, and location based system.

This paper describe a service that further extends the functionality of SMS, a prototype named as "SMS Credit" is developed that allows customers to perform electronic payment using their mobile phones via SMS, without the need to modify existing devices or to acquire new equipment. Users simply send SMS and connect to the background GSM system, key-in the charged amount, which will be automatically credited from their account once the transaction is authorized. Specific wireless terminal is installed at participating merchants and service providers, which allow customers to identify and authorize a transaction. Example scenarios on actual application on telecommunication and electronic commerce field will be discussed.
\end{abstract}

\section{Key Words}

SMS, E-payment, E-commerce, M-commerce

\section{Introduction}

Mobile phones become an essential communication tool nowadays that almost every person owns one. They are so popular nowadays, as wallets or watches, that almost every citizen in an urbanized city will carry one along with them.

GSM (Global System for Mobile communications) networks are highly developed and its coverage extends to almost every part of the city. Since any person with suitable equipment can capture information sent over radio medium, the network itself is designed to provide a secure channel for transferring digital information through the air. To achieve this, a connection must be first established and both user and background system have to mutually authenticate themselves before information is exchanged. Data is transmitted through a secure private connection afterwards and all information is encrypted using cryptographic algorithms.
With its popularity and ease-of-use, application of this secure wireless network can be further extended from a simple voice communication to other commercial areas. Mobile phones with integrated electronic purse applications are already available in the market. However, in order to access these new functionalities, customers usually have to purchase new equipments. In this paper, a prototype will be developed that allows customers to perform electronic payment using their mobile phones, without the need of modification of their mobile device or acquiring any new equipment.

Section 1 examines the current and future prospects of the mobile commerce application area. Section 2 reviews some existing mobile payment models that allow customers to perform electronic transactions using their mobile phone. Section 3 presents the framework for minipayments using mobile phones. In section 4 conclude the benefit of this mini payment for GSM operator and customer.

\section{Business Background}

With advances in wireless technologies and devices, mobile commerce is gaining its popularity and its application area is extending rapidly into different fields. According to independent research findings - $\mathrm{m}$ commerce - the conduct of business and services over portable, wireless devices - will soon dominate the business market. For example:

- IDC, an information technology research firm, reports that 70 percent of all wireless subscribers worldwide will access data applications via their phones by 2002 .

- The investment firm Robinson Humphrey predicts that, in the US, 50 percent of Internet hits will originate from wireless devices by 2004.

- The Yankee Group, a telecommunications research firm, projects that by 2004 more than 40 percent of wireless users in the US will access Internet content directly from their mobile devices.

- GartnerGroup estimates that in 2004, at least $40 \%$ of consumer-to-business e-commerce will be 
initiated from smart phones supported by WAP (Wireless Application Protocol)

- Study from the Wireless Data and Computing Services, a division of Strategy Analytics, reports that the mobile commerce market may rise to $\$ 200$ Billion by 2004 .

Internet and PC network was always been considered as the major driving force for electronic commerce. According to Yankee Group projects, the installed base of PCs will reach 500 million by 2003. These systems, with high computational power and storage capabilities, as well as low price-performance ratios, have attracted the attention of researchers and businesses from different fields to develop sophisticated electronic commerce applications.

However, PCs still have a limiting characteristic portability. Users must sit in front of them. Even in portable-notebook devices, they have to load software, dial into and connect with a network service provider, and await completion of the handshaking process before they can launch an Internet application.

This will slow down the development of electronic commerce in some particular areas, especially when dealing with mini-transactions on the business-toconsumer side. It will be quite unreasonable and unacceptable for most users to wait for a long sequence before they can perform a simple transaction.

Wireless devices are found to be suitable business-toconsumer oriented applications. According to projections of Gartner Group and other research firms, by 2004, the installed base of mobile phones worldwide will exceed 1 billion - more than twice the number of PCs. In addition, the number of other wireless mobile devices will also dramatically increase. Wireless PDA users will increase more than triple in the next years. Unlike PCs, these devices require no boot sequence and long waiting time, so people can use them as soon as they turn them on making them attractive to quick-hit applications.

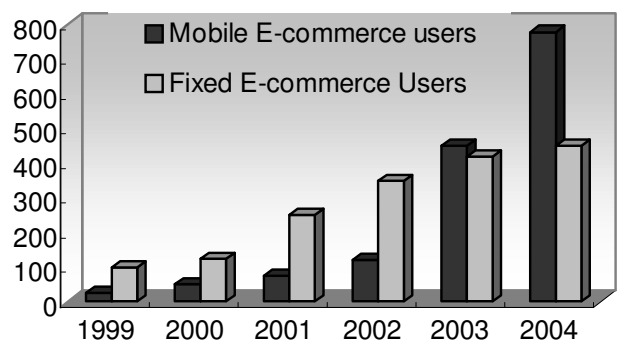

Figure 1 - Worldwide growth of e-commerce users (Source - The Arc Group)

Users will increasingly initiate a wide range of business transactions from mobile devices. They can be used for browsing and performing payments on online shopping sites. Another class of mobile commerce (denoted as mcommerce hereby) transactions involves using wireless devices to initiate and pay for purchases and services in real time. These kinds of transactions will likely increase as users gain the capability and become comfortable enough to manage them.

However, due to the bandwidth and user interface limitations, the application area of m-commerce is still restricted. M-commerce is found to fit in minitransactions field, where small amount of information is gathered from the user and exchanged with the background system. A high volume of transactions will be generated in the mini-payment areas. When e-cash equipped mobile phones or PDAs are being widely adopted to settle transactions such as subway fees - rather than coins - the use of digital cash will become a reality.

\section{Literature review}

Mobile payment systems are nowadays readily available as a commercialized product. Wireless devices can be used either as an Internet surfing tool, allowing users to perform different types of electronic transaction or as an electronic wallet that allows users to obtain resources and services from traditional distribution medium.

Numerous online shopping sites are readily available on the World Wide Web to offer different types of service and products to a wide range of customers. However, it requires a PC with Internet connection to access them. By making use of WAP (Wireless access protocol) technologies, existing Web sites contents can be translated and transmitted over wireless links and displayed on mobile devices. Service providers do not have to redesign a new website from the scratch to accommodate mobile users. Customers can shop on their favorite online shopping sites without geographical and time constrain.

Various online mobile business transactions models are developed and discussed Error! Reference source not found.2]. However, its development is constrained as it faces the traditional limitation of mobile devices: limited bandwidth, low-resolution display and a proper user interface.

Current mobile devices and technology might be more suitable for conducting electronic transactions over traditional retail medium. Nokia 6510 introduces the concept of electronic wallet, which made use of advance WAP technologies to perform electronic transactions Error! Reference source not found.4]. Siemens have introduced the concept of "pay@nce" that enables users to conduct mini-payment at registered merchants and services providers. Network operators act as a payment gateway that charges their subscribers on behalf of internal or external merchants. It makes use of SMS (Short Message Service) for transferring mini-payments from one account to another. 
A mobile wallet model is purposed on Error! Reference source not found.5], allowing existing credentials, such as bank and credit cards, be easily included within the architecture without any change to these legacy system and their functionality, thus realizing payment using offline digital cash. The authors purposed the usage of a twoslot "Subscriber Identity Module" (SIM) for implementation of the mobile payment system.

In summary, several models and prototypes were discussed on the online payment area. For electronic payment over traditional retail medium, most of these solutions require acquiring new devices or modification of existing equipments.

Telemoney offers a similar payment model allowing customer to perform their payment on registered merchant and service providers using their mobile phone (www.telemoneyworld.com).

The main idea of Telemoney is to use client's mobile as a digital identity tool to authorize online transactions performed on the Internet.

Compared to our model, it offers a similar service called Tele Cab which allows customer to pay their taxi fees by simply input a set of parameters into their mobile. Telepay is another service that allows users to pay by their mobile phones by beaming their Telemoney ID to the service terminal.

\section{Mini-payment Model}

\subsection{Motivation statement}

The diversity of applications offered by mobile phones is still restricted due to its limited bandwidth and user interface limitations. Current access technologies, including TDMA (time division multiple access), CDMA (code division multiple access) and GSM (Global System for Mobile Communication), transmit data at 9.6 to 19.2 Kbps. These speeds are dramatically slower than a normal Internet connection using a desktop PC. Although mcommerce is possible at these speeds, its development is restricted.

Unless 3G (third-generation) wireless technology is available and widely accepted by general users at a reasonable price, $2 \mathrm{G}$ (second-generation) or $2.5 \mathrm{G}$ is still the de-facto standard technology for the coming few years.

Mobile phones have a limited display and lack a userfriendly input interface. Most of the devices only offer 12digits key input. With such limitations, users will feel inconvenient when using them as surfing tool through the Internet, and a large portion of rich-content information delivered from the WWW cannot be correctly displayed. M-commerce is not still broadly adopted.
However, mobile phones are so popular that almost every person will carry one along with them. The device itself is secure enough to identify and authenticate its holder and it could be an excellent tool for authorization of credit payments. Focusing on these advantages, a prototype is designed that allows users to perform mini-payments using their mobile phones without the need of modification of existing devices.

Currently, network operators are offering value-added services to their subscribers, from download additional ring tones and logos to mobile phones, to real-time news in SMS format. The corresponding service charge for each service is deducted from the subscribers' account and they are charged upon payment of their monthly bill. These PrePaid-Services (PPS) are usually conducted internally and offered by the network operator.

In this paper, the current PPS model will further be extended, allowing external merchants and service providers to participate on this payment scheme. Network brokers or a Network operators act as a payment gateway that charges their subscribers on behalf of external participants. The payment is credited from the subscriber's account and customers are charged upon payment of their monthly bill.

Similar to credit card acquirers, Network Operators (NO) or Network brokers (NB) comprises the role of a credential company that will pay in advance to merchants and service providers on behalf of their subscribers. NO or NB can either charges their subscribers for using these services via their billing infrastructure, or deduced the corresponding amount upon payment to external service providers. There are other advantages for NO over a usual acquirer:

- $\quad$ Reduced risk of bad debt

- Immediate payback of service usage

- $\quad$ Lower costs of the NO

- $\quad$ Easy access of new subscribers to NO services

- Access to most updated subscriber information

The most noticeable advantage of the NOs own is their financial customer relationship. They send a monthly bill to their subscribers and might even know their account data at financial institutes. Their financial risks are much lower than usual acquirers. On the other hand, NOs could charge service providers for using their billing infrastructure, as they are offering an added value service to the merchant which it is worth to pay.

\subsection{Design requirements}

The main requirement of this project is to develop a minipayment model using mobile phones without the need of modification of existing wireless devices. The model is designed to allow consumers to conduct electronic payment by simply key-in several codes via Shot Message 
Services (SMS) to specific MSISDN (Mobile Station ISDN) using the 12-keys input pad available in most standard mobile phones.

Furthermore, customers expect an efficient payment solution when dealing with low value transactions. Standard macro-payment or electronic cash protocols make use of cryptographic algorithms to achieve security and privacy. Transactions must be authorized by a central independent third party. This results in a central bottleneck on most of the on-line payment schemes, a single point of failure that increases payment latency. It also raises the cost of the transaction, and imposes a minimum cost per transaction, as the bank is faced with the real cost of authorizing each transaction. Error! Reference source not found.3]

In this payment model, Network Operator will act as a central authority that handles all the transactions. They will charge on behalf of the merchant and vendors and credit the customer's account once the transaction is completed. GSM network architecture provides mechanism for authentication of user and encryption of transmitted messages. As it is dealing with low value transactions, payment information will be encrypted using the standard cryptographic algorithms offered by the GSM Network. To minimize the risk of payment using stolen or loss mobile devices, users have to authenticate themselves using a PIN code for authorizing each transaction.

Privacy cannot be achieved in this model and users are expecting to reveal their identity. As in credit card payment models, users have to identify themselves using a card and the corresponding account is credited. In our model, users are identified by their MSISDN.

\subsection{System overview}

\section{Assumptions}

1. The billing architecture of the Network operator could be extended to handle external financial transactions.

2. Security of messages transmitted through radio communication network is achieved by using cryptographic services offered by the GSM Network.

3. Users are identified by their MSISDN.

4. Background operation of the billing system will be out of scope of this paper.

This model requires network operators to offer additional services more than the existing voice-call system. Traditionally, NOs provides basic telecommunication services (voice-calls, SMS, etc.) that charge their subscribers according to their usage. In our model, NOs have a new role: they act as a Payment Service Provider (PSP) that charges their consumer on behalf of external merchant for purchases of a product via their billing infrastructure.

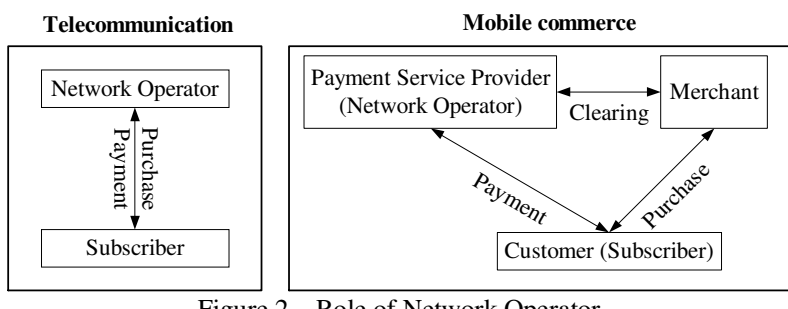

Figure 2 - Role of Network Operator

Each subscriber's account does not only represent the amount charged for usage of telecommunication services offered by the NO, it also accounts and credits the consumer for any m-commerce purchases and services initiated using their mobile devices. As a consequence the NO who is willing to participate in this business has to modify his payment infrastructure in order to accept new internal and external merchants.

Authorization terminals can be installed on participating merchant and service providers. These are special GSM devices that are designed to allow consumers to confirm and authorize a particular transaction.

In order to access this payment service, customer must be a valid mobile subscriber of the network operator, and he/she must have applied short code messaging service and registered a Mobile payment user".

The following figure shows how the model works:

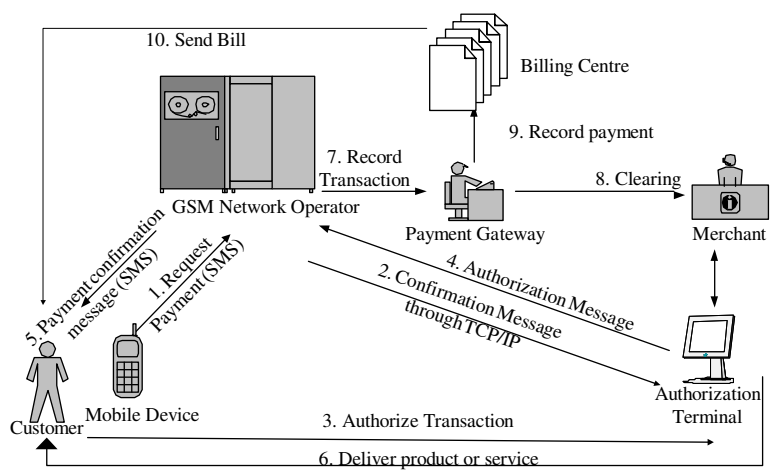

Figure 3 - Mini-payment model with authorization terminal

1. Consumer sends SMS to the Payment Gateway. A set of parameters is key-in using their mobile device, separated with the special key "\#”: vendor's code, their secret PIN and the corresponding amount to be charged or key-in using their mobile device, separated with the special key "*": Product short code, their secret PIN. A confirmation message through TCP/IP is sent to the merchant's authorization terminal. Consumer's mobile number and amount to be charged are shown on the terminal for authentication. 
2. Consumer authorizes the transaction by key-in a second PIN code in the through authorization terminal.

3. Authorization terminal sends a message back to the Network Operator along with the secret PIN code to confirm that the customer has authorized the transaction.

4. Consumer receives a SMS message for notification of payment completion.

5. Merchant or service provider delivers the product or service to the customer.

6. The transaction is recorded on the Payment Gateway server.

7. Merchant can request for clearing through the Payment Gateway.

8. Payment Gateway sends transaction records to the billing center.

9. The corresponding credited amount is recorded and included in the monthly bill and sent to the subscriber.

The functionality of the model depends on the type of service or product offered to the customer, which is directly related to the value of the transaction. In the last scenario, an authorization terminal must be installed on participating merchant or service providers. This will increase the cost of each transaction. When dealing with lower value transactions, customer will face lower risks on both security and financial point of view. To further reduce the transaction cost, a second scenario is presented where authorization terminal is removed from the previous model.

The following figure presents another possible scenario that does not require an authorization terminal to be installed on the merchant's site.

1. Consumer sends SMS to the Payment Gateway. A set of parameters is key-in using their mobile device, separated with the special key "**”: vendor's code, their secret PIN and the corresponding amount to be charged.

2. GSM Network Operator records the transaction by the payment gateway server

3. Consumer receives a SMS message for notification of payment completion.

4. Merchant receives a SMS message through his mobile device for notification of payment completion.

5. Merchant or service provider delivers the product or service to the customer.

6. Merchant can request for clearing through the Payment Gateway.

7. Payment Gateway sends transaction records to the billing center.

8. The corresponding credited amount is recorded and included in the monthly bill and sent to the subscriber.
In this model, merchant have to trust that network operator has credited the payment amount from the customer. They have no means to assure that the required amount is charged until they payment is cleared.

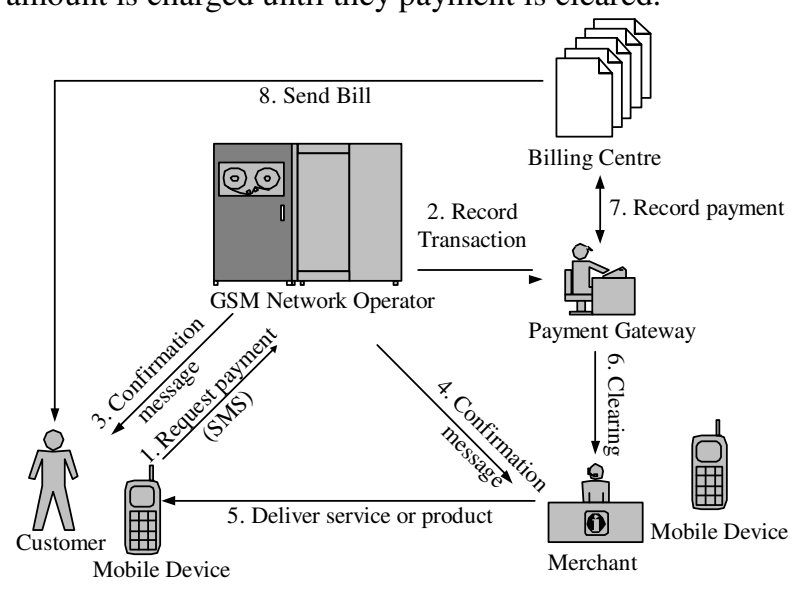

Figure 4 - Mini-payment model without authorization terminal

\subsection{Detailed payment model}

The purposed payment model has just discussed in a simplified form. The actual payment model involves interaction with the GSM background system. Details on how users are authenticated and messages are encrypted by using the services offered by the GSM network does not discussed.

In summary, the process is shown as follows:

1. Customer chooses a valid short code of a merchant and keys in the short code in SMS and sends out. The request will be transferred to the BTS through radio, and finally transferred to MSC through the BSC.

2. The GSM network authenticates customer by a service table, which is located in the HLR (Home Location Registers) related to the MSC. It will check the request from the customer (mobile phone short code) with its short code table and find the matching in the service located in the HLR.

3. If this short code and service belongs to the mini payment service, it will route the request to the IVRS (Interactive Voice Response System), which has been integrated as part of the VAS (Value Added System).

4. After the VAS received the request, customer key in its secret PUN and the system in return will either accept the customer's request by sending a confirmation "voice message" or denied the customer with a rejection "voice message". 
5. Customer request will be routed to the payment gateway, which will authenticate the request with both merchant and customer billing center.

6. The SMSC (Short Message Service Center) will to merchant for requesting final authorization from the customer

7. Merchant terminal will display the authorization requested message for final confirmation.

8. If customer agrees with the transaction, a short message will be send back to the SMSC, which will route the message to the payment gateway.

9. The billing account of customer will be credited, and the merchant account will be debited if customer agreed with the transaction.

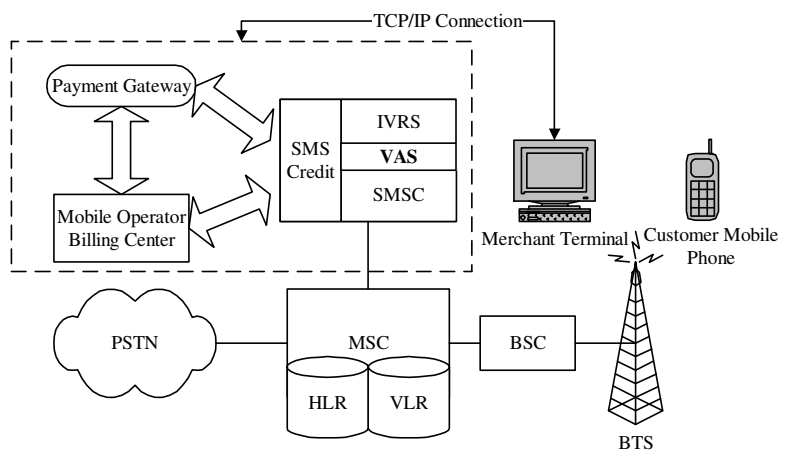

Figure 5-GSM System overview

\section{Software prototype}

\subsection{Actors}

Three different actors are identified in this system, as shown in Figure 6 . They could be briefly described as follows:

1. Phone Client- these are the customer of mini payment system. They could request the payment service by sending SMS and authorize the transaction by key in their own PIN code.

2. Merchant - a potential Phone Client that can request service and receive payment. Merchant can receive his own on merchant's sites transaction verification and authorization. Merchant will deliver the related product or service to Phone Client once the payment transaction has been accepted and authorized successfully

3.Server Administrator-the system user make use of background system which responsible to process the customer and merchant to request and receive payment service respectively, triggering SMS and confirmation or rejection message. They are also responsible for maintaining the Phone Client and Merchant information, status and have the ability to detect system fraud.
Administrator users are also allowed to request service and receive payment in the mini payment system
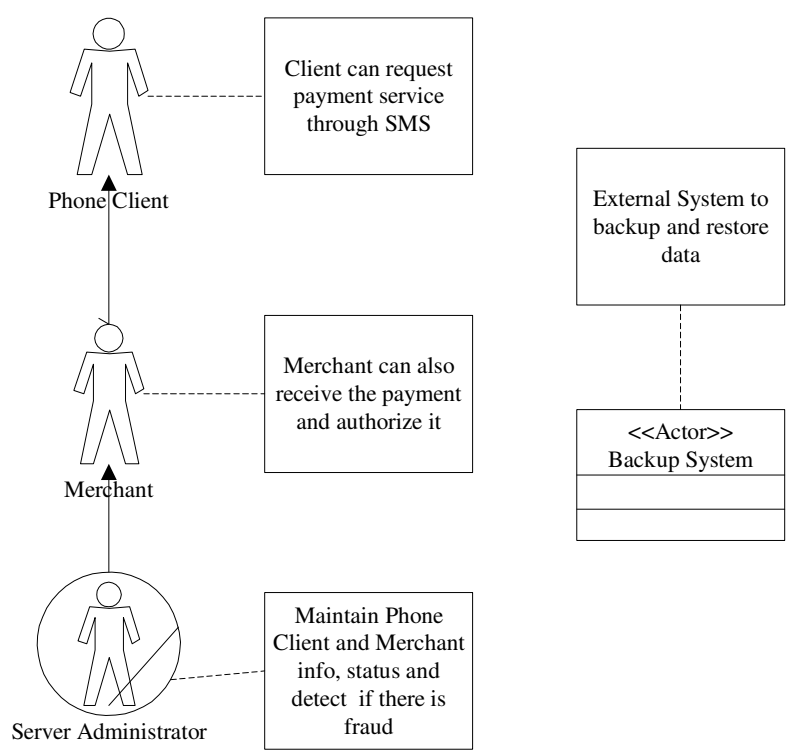

Figure 6.User relationship in the mini payment system

\subsection{Use Case Diagram}

Figure 7 shows a Level-0 user-case diagram of the mini payment system, which gives an overview of the requests of each actor:

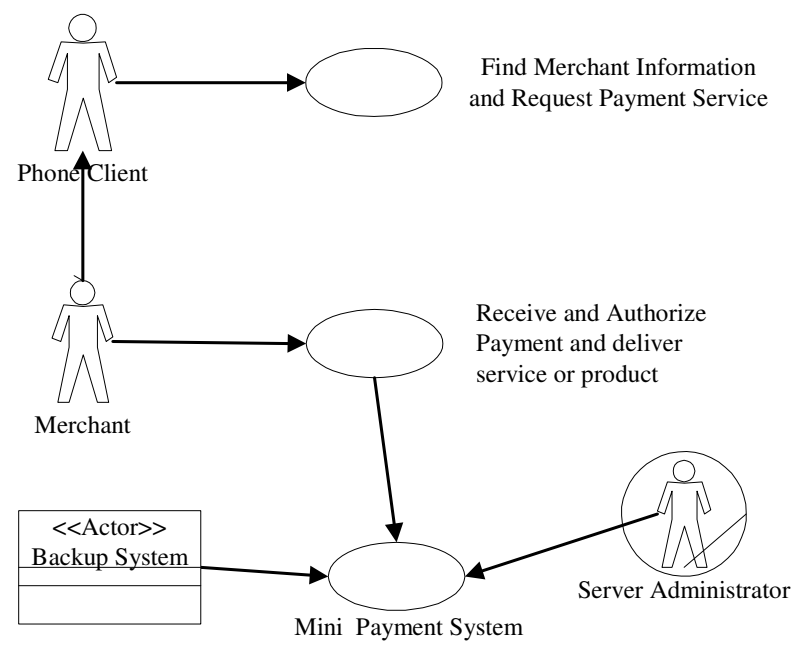

Figure 7. Level 0 User-case diagram

- phone client is the mobile subscriber which is registered in the network operator payment system and can request payment service

- Merchant is an extension of the phone client. In addition to the basic functionalities available to the phone client, merchant is allowed to receive and authorize payment and request for clearing through the payment gateway.

- Server administrator is responsible for maintaining the Phone Client and Merchant information, status 
and has the ability to detect system fraud. System users are also allowed to request service and receive payment in the mini payment system

- The backup system is an external actor that will contribute interactively in the maintenance of the system recovery procedures.

Level-0 diagram gives only a brief overview on how each actor will interact with the system. A more detailed analysis will lead to Level-1 diagrams, which will describe in more detail the individual requirements of each actor.

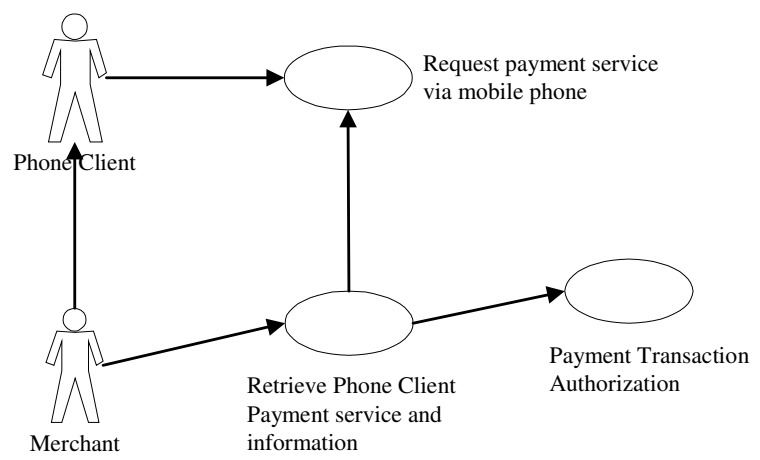

Figure 8- Level 1 User-case diagram- Phone Client

A phone client is a user of the mini payment system. The mini payment system will only provide service for this registered user. Merchant is a potential phone client that could access to the main payment functions of the system. They have access to accept or reject transaction of a payment request, deliver an item to phone client once transaction is accepted and authorized successfully. The librarian accesses these functions on behalf of the borrower.

Server Administrator is the actor responsible for the maintenance of the payment service between phone client and merchant. Once there is payment failure, the administrator has the ability to check and correct it. The administrator has the permission to access all the functions carried out by the payment system, and they are responsible in maintaining the phone client and merchant information, status and interact with the backup-system

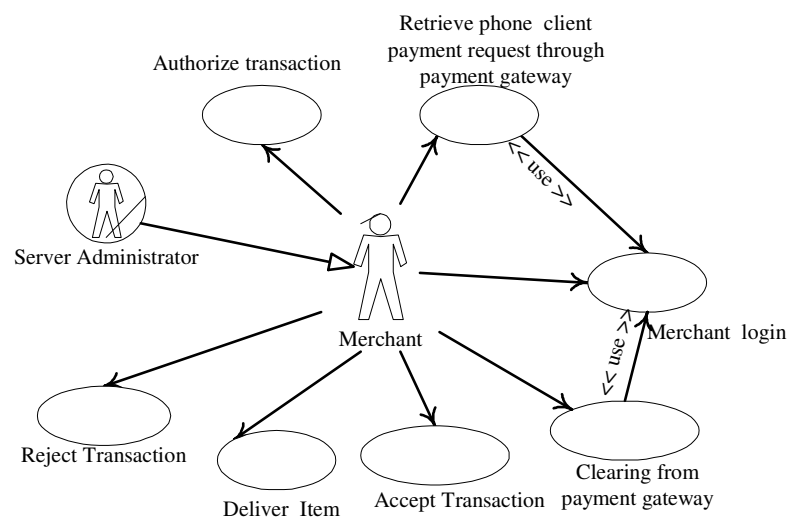

Figure 9. User-case diagram - Merchant

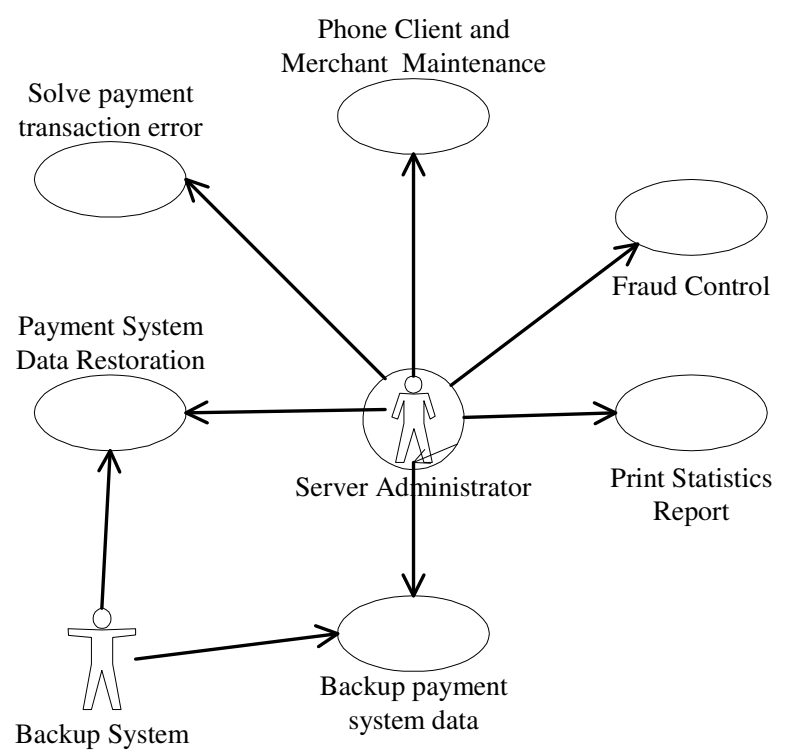

Figure 10. User-case diagram-Server Administrator

\subsection{Class Diagram}

Class diagram is created to provide a picture or view of some of the classes in the model. It describes the relation between the entities which interact in the system. The class diagram that describes the entity in the mini payment system.. As shown in Figure 11 the diagram shows the database structure for the payment system. It shows the database structure for the library system. From the diagram, the Merchant is inherited from the Phone Client, and the Server Administrator is inherited from the Merchant.

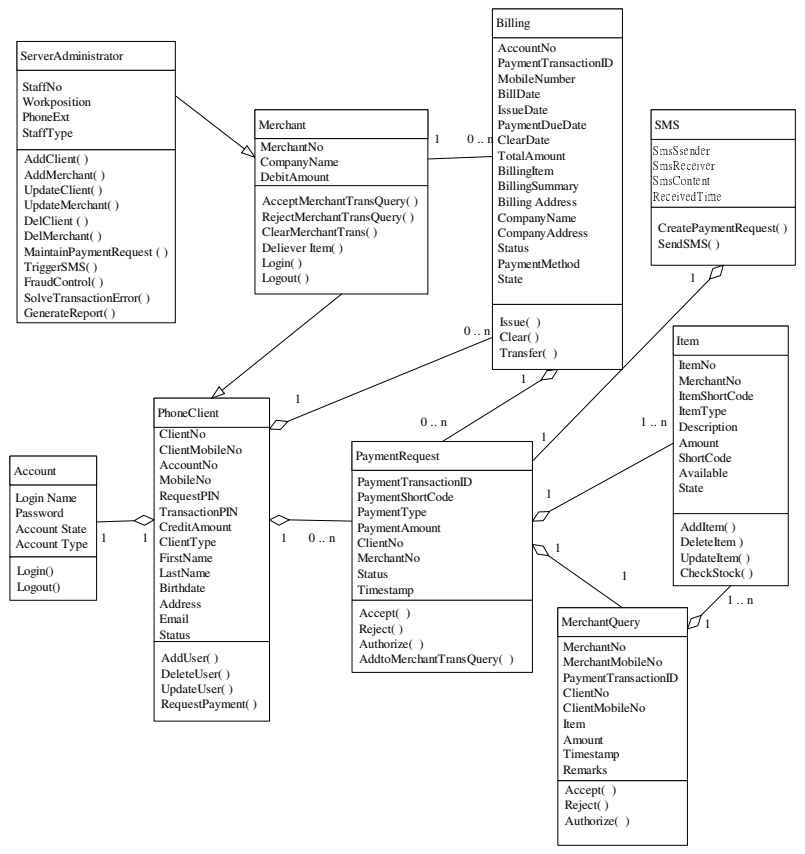

Figure 11 Class Diagram 


\subsection{Sequence Diagram}

With proceed with the dynamic behavior aspect of the analysis. Sequence diagram of the phone client will be shown. A sequence diagram for the use case "Request Payment Service" is shown in Figure 12.

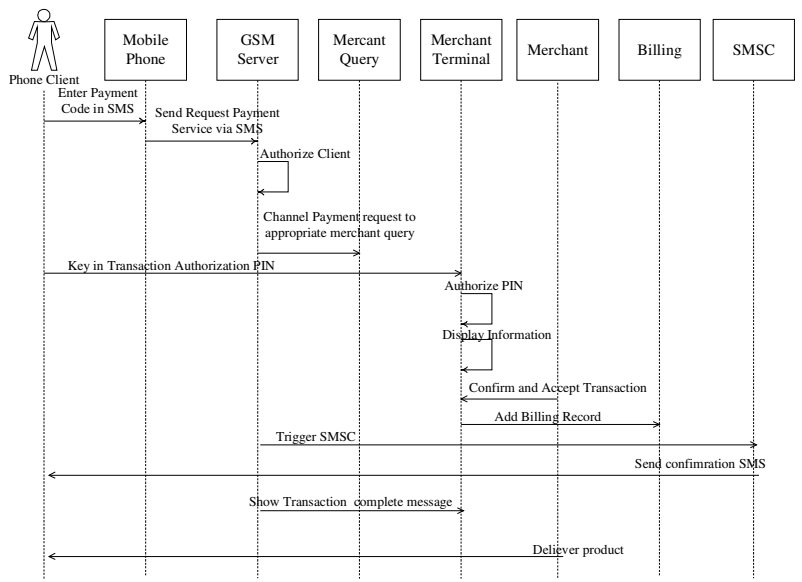

Figure 12- Sequence diagram for Phone client request payment service

\subsection{Activity diagram}

Activities diagram describe the activities of a class. They describe the behavior of a class in response to internal processing rather than external events. Activity diagram about phone client request payment with Merchant Terminal will be shown.

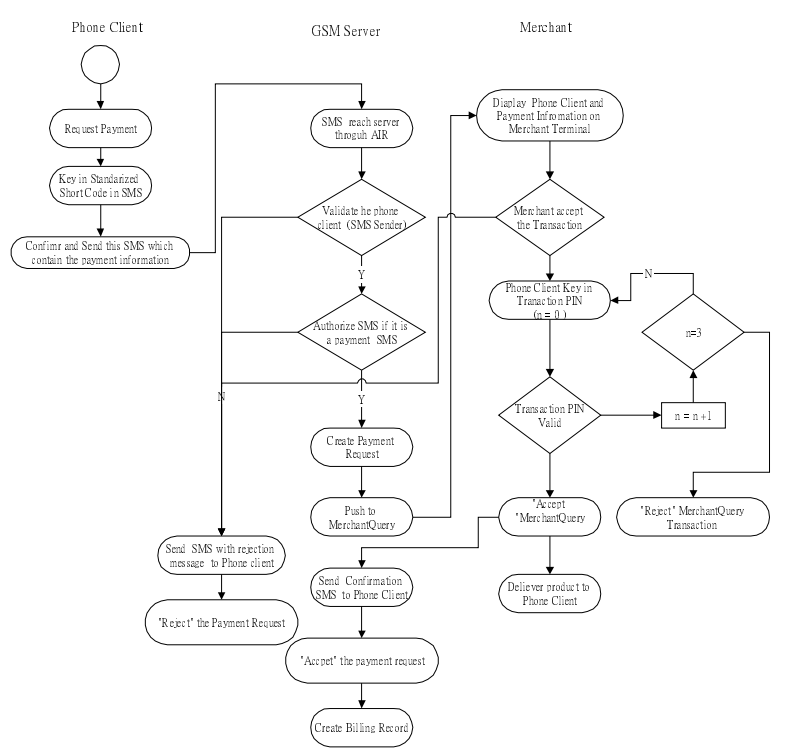

Figure 13. Activity Diagram of Phone client request payment with Merchant Terminal

\subsection{Implementation}

Programming begins during the construction or implementation phase. The component and deployment diagrams, which describe the organization of and dependencies among software implementation components, and the configuration of processing resource elements and the mapping of software implementation components onto them, respectively are shown in the following:

In this software prototype, there are three components

1. Phone Client is only a mobile phone or a mobile device which emulates the basic functionalities of a mobile client acting as a wireless payment device.

2. GSM Server emulates the background system that serves as a payment gateway recording each transaction. This GSM server is composed with a mobile phone which is used to retrieve the SMS of Phone Client Request

3. Merchant terminal emulates the device installed on merchant's sites for transaction verification and authorization.

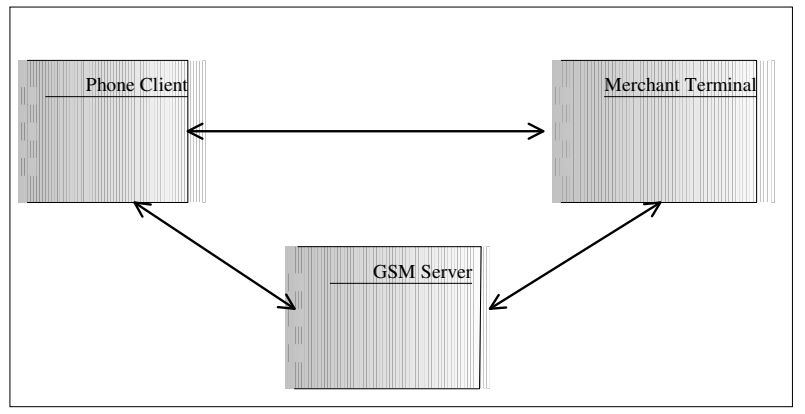

Figure 14. Component diagram

The payment model is simplified by making the following assumptions:

- Only the basic functionalities required to demonstrate the payment model are implemented.

- Communication between components is simulated using TCP/IP protocol.

- Messages are transmitted in plain-text format.

\subsection{Phone client}

Phone client mainly emulates the basic functionalities of a mobile device used as an electronic payment tool on the model purposed in the last section. By using the 12-keys input pad offered on most mobile phones, user input a set of string via SMS for initialization of a payment.

In order to be correctly interpreted by the GSM server, the string must have a standardized format. In this minipayment, there are two payment services offered: 1) credit transfer and 2) short code payment

1) Credit Transfer-The billing account of customer will be credited with this specific payment amount, and the merchant account will be debited if customer agreed with the transaction.

\section{\#[Merchant ID]\#[User PIN]\#[Payment Amount]\#}


The decimal point is represented by \#. In the following example, the amount of $\$ 88.80$ is represented by $88 * 8$.

2) Short Code Payment-Customer only need to key in the Short Code for requesting the product or service. The payment amount charged based on Merchant Service or Product Code Table.

\section{* [Product or Service Short Code]*[User PIN]*}

In this model, after the user input the whole payment string, user must click on the SEND button in order to transfer the SMS message to the GSM Server. TCP/IP is used to simulate the communication protocol between the merchant terminal and the background GSM server. The server is listening to the port 1007 to receive messages transmitted from the merchant terminal.

As discussed before, information transmitted through the GSM network is secured and messages transmitted through the air are encrypted when a session is established with the background system. The unique PIN number contained in the message cannot easily be eavesdropped by attackers.

\subsection{GSM Server}

GSM server primarily emulates the background system connected to the GSM network, the billing system and payment gateway. The basic functionalities are:

1. Listen to client payment requests.

2. Identify and authenticate the client.

3. Send authorization confirmation messages to merchant terminals.

4. Record transactions and transfer it to the billing system.

When the server receives the payment message, the interpreter will decode it and perform the following checks:

1. Locate the customer from the database by its Customer ID. An error message is returned if the corresponding ID does not exist on the database.

2. Locate the merchant from the database by its Merchant ID. An error message is returned if the corresponding ID does not exist on the database. If match is found, the corresponding port number is retrieved from the database.

3. Finally, the PIN of the customer is retrieved from the database and compared with the one received from the user. If they do not match, an error message is returned.

The transaction proceeds if the correct PIN is matched. The message is sent to the corresponding merchant terminal according to their port number.

In the payment system, the merchant service query is updated through TCP/IP connection or a SMS message is sent to the merchant terminal, which is a specialized designed GSM client similar. This device is designed to retrieve merchant service query or received SMS messages transmitted from the GSM server and act as an authorization terminal where customers are able to confirm their transaction.

\subsection{Merchant Terminal}

Merchant terminal models the special designed GSM client used to authorize a transaction. It will listen to payment confirmation messages by TCP/IP sent from Server or from GSM servers in SMS format.

When a message arrives, it will display the corresponding client information in the terminal screen, along with the amount to be paid. User can select to accept the transaction of reject it.

If the user chooses to accept the transaction, a confirmation message is sent back to the GSM server. When the message arrives to the payment gateway, the transaction is recorded in the database and later will be sent to the billing system for processing. The server will finally send a payment receipt in SMS message format to the client's mobile to acknowledge the transaction.

If the user chooses to reject the transaction, a reject message is sent back to the server and the transaction will be aborted.

\subsection{Deployment Diagram}

Deployment diagram describe the configuration of the processing resource elements and the mapping of software implementation components onto them. The deployment diagram Figure 15 shows that the phone client will execute payment service on a mobile phone. The Merchant accesses the system from the Merchant. The solid paths represent lines of communication between nodes which is directly connected. SMS is trigged by using the API provided in Web server. The dash path represents lines of communication through air interface.

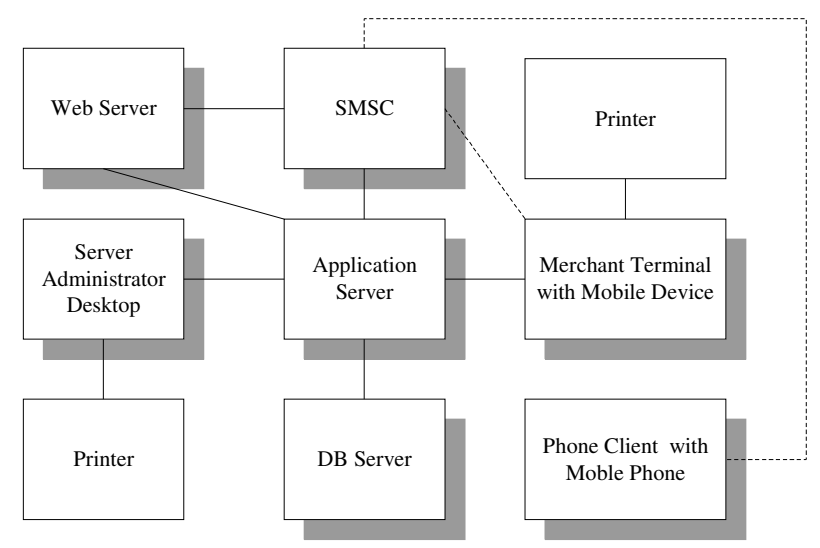

Figure 15. Deployment diagram 


\section{Sample Source Code}

In the following, the programming code on how the SMS is sent and retrieve is discussed. In this software prototype, a SMS modem has been employed to interact with incoming and outgoing SMS. All incoming SMS will be stored in place, which is provided for the application server to check if the SMS is in payment SMS format. Outgoing SMS can be sent through HTTP post in dedicated web server. This model can be further developed to include peer-to-peer payment, allowing two users to exchange money directly through their mobile phones. For instance,

http://smscredit/?Phonenumber=98989898\&Text=sample

On the other hand, a SMS gateway has been employed and it is provided by one of the IT company [9] which has a corporation with Network operator. The connection method is HTTP / HTTPS- submitting either a POST or GET to the API server. The following figure shows one of the simpler server-based forms of communication to the API. It can be used either in the form of a HTTP POST, or as an URL (GET). POST method is recommended for larger data transfer due to the size limitations of GET. Communication to the API can be done either via HTTP on port 80 or HTTPS on port 443. All calls to the API must be URL-encoded. The parameter names are casesensitive.

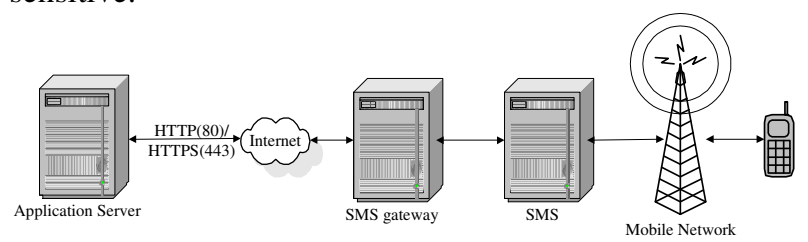

Figure 16.Block Diagram of HTTP/HTTPS API Infrastructure

For instance,

1. Here is an example to send an English SMS.

https://www.bbsend.com/api/servlet/SendMessage?userna me $=$ bbsend $\&$ password $=123456 \&$ unicode $=0 \&$ receiver $=98$ $989898 \&$ message $=$ sample

2. Here is an example to send an Unicode SMS.

https://www.bbsend.com/api/servlet/SendMessage?userna me $=$ bbsend $\&$ password $=123456 \&$ unicode $=1 \&$ receiver $=98$ $989898 \&$ message $=6$ B618FCE4F7F752800200062006200 730065006E0064002E0063006F006D00200053004D005 3002000470061007400650077006100790020670D52D9

3. Here is an example to retrieve message.

https://www.bbsend.com/api/servlet/GetMessage?userna $\mathrm{me}=$ smscredit $\&$ password=123456\&action $=$ unread

\section{Conclusion}

GSM network can provide a strong security for each mobile subscriber, and it is found to be a suitable medium for performing off-line transaction of small amount.

A model has been developed allowing GSM subscribers to effectuate payments by their mobile phones without need of modification of existing equipment or acquiring new equipments. Users simply key in a set of parameters to initialize a payment to registered merchants or service providers, and they account will be charged accordingly. Network operators will act as acquirers to credit customer's account to pay-in-advance to merchants they deal with.

This value-added service offered by network operators helps them to increase their market share as they are offering a much more convenient payment option to their subscribers.

\section{References}

[1] The Emergence of M-Commerce - James A. Senn Pages 148 - 150

[2] A Framework for the Emerging Mobile Commerce Applications - Upkar Varsney, Ron Vetter

[3] Evolution of Prepaid Service towards a Real-time Payment System - Manfred Lilge

[4] Nokia 6510 official website http://www.nokia.com/phones/6510/index.html

[5] Siemens Mobile Payment official website http://www.siemensmobile.de/pages/payment/en/index.html

[6] Localized Credentials for Server Assisted Mobile Wallet - Stig Frode Mjolsnes and hunming Rong

[7] Comparing and contrasting mini-payment models for E-commerce systems - Xiaoling Dai, John Grundy and Bruce W N Lo

[8] www.telemoneyworld.com - Telemoney Mobile Payments website

[9] http://www.bbsend.com

[10] GMS 07.05, GSM Technical Specification ETSI

[11] Extended ETSI Hayes AT command parameters for SMS-http://www.cellular.co.za/at_etsi.htm

[12]GSM World - What is SMS http://www.gsmworld.com/technology/sms/intro.sht $\mathrm{ml}$ 